\title{
Flexible and Wearable GRF and EMG Sensors Enabled Locomotion Mode Recognition for IoHT Based In-home Rehabilitation
}

\author{
Chaoming Fang ${ }^{1}$, Yixuan Wang ${ }^{1}$ and Shuo Gao ${ }^{1,2, *}$ \\ ${ }^{1}$ School of Instrumentation Science and Optoelectronic Engineering, Beihang University, Beijing, China \\ ${ }^{2}$ Beijing Advanced Innovation Center for Big Data-Based Precision Medicine, Interdisciplinary Innovation Institute of Medicine \\ and Engineering, Beihang University, Beijing, China \\ *shuo_gao@buaa.edu.cn
}

\begin{abstract}
Benefiting from the development of the Internet of Healthcare Things (IoHT) in recent years, locomotion mode recognition using wearable sensors plays an important role in the field of in-home rehabilitation. In this paper, a smart sensing system utilizing flexible electromyography (EMG) sensors and ground reaction force (GRF) sensors for locomotion mode recognition is presented, together with its use under the IoHT architecture. EMG and GRF information from ten healthy subjects in five common locomotion modes in daily life were collected, analyzed, and then transmitted to remote end terminals (e.g., personal computers). The data analysis process was implemented with machine learning techniques (Support Vector Machine), through which the locomotion modes were determined with a high accuracy of $96.38 \%$. This article demonstrates a feasible means for accurate locomotion mode recognition by combining wearable sensing techniques and the machine learning algorithm, potentially advancing the development for IoHT based in-home rehabilitation.
\end{abstract}

Keywords-Flexible sensors; Locomotion mode recognition; Internet of Healthcare Things; In-home Rehabilitation

\section{INTRODUCTION}

It is of great significance to monitor the physical information, such as gait and muscle condition, of patients recovering from injuries or diseases under different locomotion modes to make a proper rehabilitation plan and evaluate the rehabilitation effect [1-5]. Tradition scenarios to obtain these data are mainly located in hospitals or therapy institutions $[6,7]$. However, patients may suffer pressure when being monitored, resulting in low quality data, conversely influencing the evaluation of the rehabilitation effect [5].

In recent years, with the fast development of wearable electronics and the Internet of Healthcare Things (IoHT) techniques, in-home rehabilitation has emerged quickly and gained worldwide attention, as in-home rehabilitation not only alleviates patients' undesired stress but also is capable of collecting high volumes of useful data by conducting long-term monitoring [8-10]. For in-home rehabilitation, locomotion mode recognition is important, which is closely related to the interpretation of body signals picked up by wearable systems [5, 11-13]. Hence, locomotion modes are must-have information delivered to hospitals under an IoHT architecture.

A locomotion mode recognition system generally consists of two parts: a data acquisition front-end and a recognition algorithm back-end. For the former, wearable sensors, including kinematic sensors (IMUs) [14-16], kinetic sensors (interaction force or ground reaction force sensors) [17], and electromyography (EMG) sensors [16-19] are widely used. For the latter, machine learning is frequently used for analysis of the physical information received from the front-end. For example, in [19], EMGs from nine muscles and six GRF measurements from load cells were collected, and Support Vector Machine (SVM) and Linear Discriminant Analysis (LDA) were used to identify five locomotion modes continuously with an accuracy no lower than $93.44 \%$. In [16], seven EMG and two accelerometer sensors were used, and five locomotion modes were classified by SVM and LDA, with an accuracy of $95.2 \%$. In [17], EMG, GRF, interaction force and position sensors were integrated to classify five locomotion modes with Bayesian LDA and reached an accuracy of $96.1 \%$.

However, the successful broad use of locomotion mode recognition for in-home rehabilitation has not been reported yet. A possible explanation is that the utilization of multiple sensors not only increases the overall component cost, circuitry complexity, and energy consumption, which are undesirable factors for electronic products, but also brings users inconvenience when used. To address this, in this article, we present a smart locomotion mode recognition system with a high experimentally demonstrated classification accuracy of $96.38 \%$ by utilizing only two EMG and two GRF flexible sensors and a machine learning algorithm. Compared to the previous studies [14-19], the developed technique benefits from simplicity by smartly designing measurement locations and selecting highly correlated features for the machine learning algorithm while maintaining a high detection accuracy.

\section{METHODOLOGY}

\section{A. Experimental Protocol}

The predefined locomotion modes were identified as follows: (1) level walking (LW), (2) ramp ascent (RA), (3) ramp descent (RD), (4) stairs ascent (SA), (5) stairs descent (SD). The above locomotion modes are consistent with those in other relevant works [16-19]. Ten healthy subjects ( 6 male and 4 female) were asked to walk using the five locomotion modes and ultimately produced 3,573,241 observations, with 2707 strides in total. The ramp was angled at 10 degrees, and the stair height was $10 \mathrm{~cm}$ [17].

This work was supported in part by the National Natural Science Foundation under Grant 6180301, and in part by the Beihang University under Grant KG12090401 and Grant ZG216S19C8. 


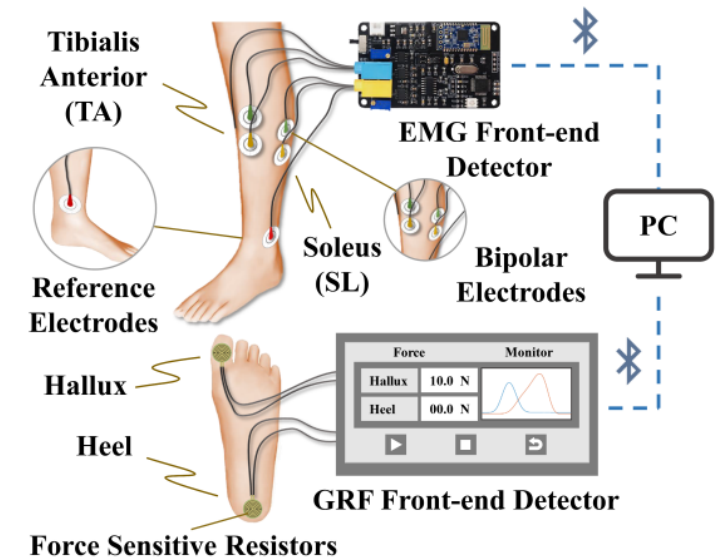

Fig. 1. The sensors locations and an overview of front-end EMG and GRF signals detection.

\section{B. Flexible Sensors and Readout Architecture}

The setup of front-end system is conceptually depicted in Fig.1. The flexible EMG sensor is a $10 \mathrm{~mm}$-circular gelled electrode utilizing electrolytic gel as the interface between the skin and the metallic part of the sensor, whose composition is silver-silver-chloride $(\mathrm{Ag}-\mathrm{AgCl})$ [20]. Three electrodes were used to detect the EMG signal for a muscle with bipolar electrodes attached to the surface of the muscle and a reference one attached to the ankle [21]. Tibialis Anterior (TA) and Soleus (SL) were selected as the detected muscles and therefore six electrodes were used for EMG detection. Two flexible Force Sensitive Resistors (FSR) were attached to the flat area of the heel and hallux to collect the GRF signal.

The EMG and GRF signals were read out, preprocessed, and transmitted to a PC, as shown in Fig. 2. The raw EMG generated by the muscle was amplified by differential amplifiers to suppress the common mode input. An analog-to-digital convertor (ADC) and bandpass digital filtering $(10 \mathrm{~Hz}-500 \mathrm{~Hz}$ [20-22]) was followed by the embedded MCU. For GRF signals, the resistance change of the FSR was linearly converted to a voltage change by a proportional operational amplifier and then converted to a digital signal by an ADC. Both the digital EMG and GRF signals were then conveyed to two Bluetooth devices (Huicheng, China) by the Serial Protocol and transmitted wirelessly via Bluetooth to PC. The major parameters of the front-end data detection system are summarized in Table I.

\section{Locomotion Mode Recognition Algorithms}

To recognize the locomotion modes of the user, a recognition algorithm using EMG and GRF signals from the front-end has been developed through three steps, as shown in Fig.3. Firstly, the EMG and GRF signals are preprocessed. All the datapoints are synchronized by timestamp. Normalization is performed to reduce the variability of each stride [23, 24], and the signal sequence is segmented into each stride by a binarized GRF signal. Secondly, twenty-one statistical features, like the time domain features of EMG [25] and the force/time information of GRF, are extracted from the segmented EMG and GRF sequence in each stride. Finally, the extracted features were used to train the machine learning model, i.e., the SVM model, and then the model was used to predict the locomotion mode. A 10 -fold cross validation was used to evaluate the accuracy of the

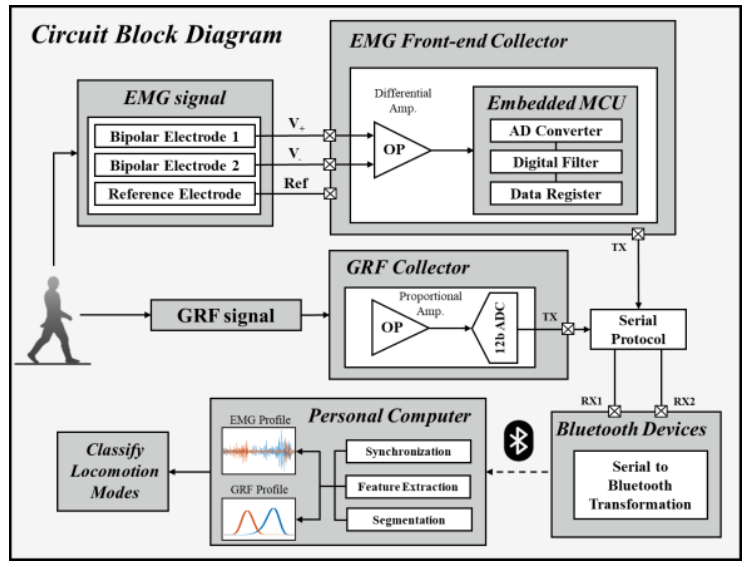

Fig. 2. The block diagram of the circuit and devices for detecting, processing and transmitting EMG and GRF signals.

TABLE I. SUMMARY OF THE MAJOR FRONT END SYSTEM PARAMETERS

\begin{tabular}{|c|c|c|c|}
\hline \multicolumn{4}{|c|}{ System Parameters } \\
\hline \multicolumn{2}{|c|}{ GRF } & \multicolumn{2}{c|}{ EMG } \\
\hline ADC Resolution & $12 \mathrm{Bit}$ & ADC Resolution & $12 \mathrm{Bit}$ \\
\hline Data Rate & $115,200 \mathrm{bps}$ & Data Rate & $115,200 \mathrm{bps}$ \\
\hline Sampling Rate & $100 \mathrm{~Hz}$ & Sampling Rate & $1 \mathrm{kHz}$ \\
\hline Response Time & $10 \mathrm{~ms}$ & Passband & $10-500 \mathrm{~Hz}$ \\
\hline Triggering force & $0.2 \mathrm{~N}$ & Magnification & 500 \\
\hline
\end{tabular}

trained model [26]. The above procedure was carried out in the MATLAB software and the classified locomotion mode together with the EMG and GRF profiles can be displayed in this software.

\section{RESULTS AND DISCUSSION}

The constructed wearable system is employed for recognizing users' different locomotion modes. A typical profile of the EMG amplitude and normalized GRF stress values under different locomotion modes is demonstrated in Fig.4. This figure demonstrates that the EMG and GRF profiles vary from each other under different locomotion modes. For example, when walking on level ground, the main activities of SL occur at about $50 \%-60 \%$ of the gait, and the heel will contact the ground prior to the hallux, as shown in Fig.4 (a), (f). In contrast, Fig.4 (e), (j) shows that SL is most active at about $10 \%$ of the stride, and

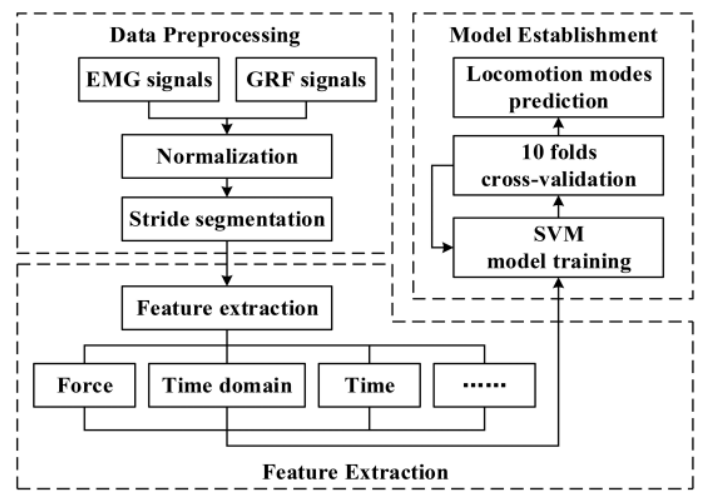

Fig.3. Flowchart of locomotion modes recognition algorithms based on EMG and GRF signals 

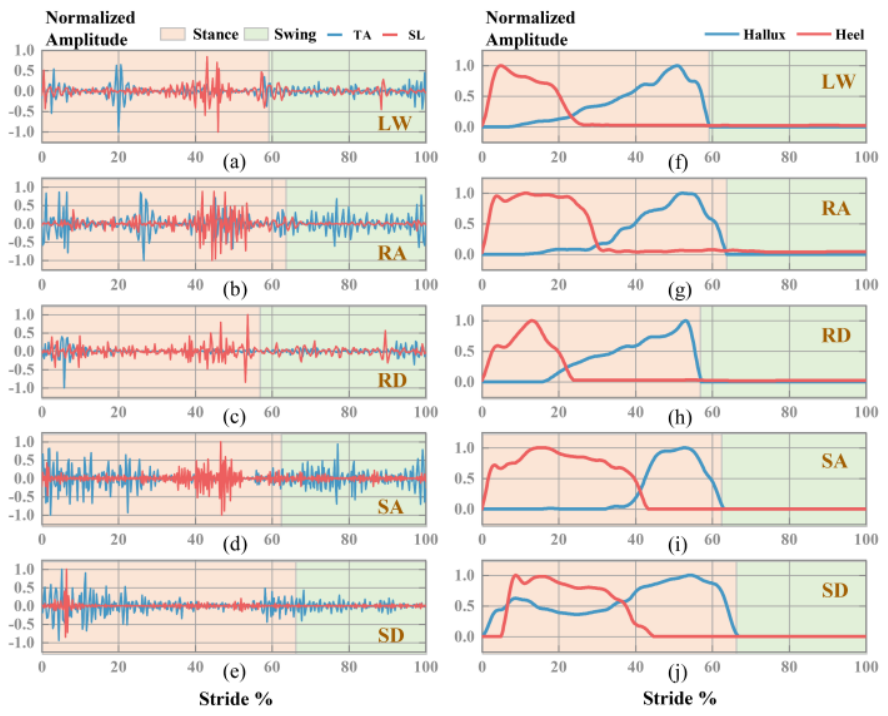

Fig.4. Typical profiles of EMG (left column) and GRF (right column) in five locomotion modes: (a), (f) level walking (LW). (b), (g) ramp ascent (RA). (c), (h) ramp descent (RD). (d), (a) stairs ascent (SA). (e), (j) stairs descent (SD).

conversely, the hallux contacts the ground first when walking downstairs. This is because the front foot must reach out first, and the SL should contract in order to maintain balance in such cases. The profiles in Fig.4 indicate that the muscle activities and gait information were different in each mode in order to adapt to the relevant terrain. However, the profiles are sensitive to many uncontrollable factors, such as changes in the bodily conditions of the user. It would be difficult to recognize different locomotion modes by simply evaluating a few metrics with some fixed thresholds. Therefore, machine learning method is applied to comprehensively evaluate the non-linear changes of the EMG and GRF inputs to obtain robust recognition

Here, an SVM model was trained and tested with extracted features. Fig. 5 shows the confusion matrix of the model. An average accuracy and variance of $96.38 \%$ and $1.08 \%$ was obtained by the trained model. This model achieved the best recognition performance in recognizing $\mathrm{SD}$, with the highest accuracy of $99.64 \%$ among all modes. This is because the EMG and GRF profiles of SD are rather unique and thus are easier to classify. However, the performance when recognizing some modes of the model, like distinguishing $\mathrm{LW}$ from $\mathrm{RD}$, is relatively lower and less robust, with an error rate at $5.04 \%$ and a variance of $3.49 \%$. A possible explanation for this is that, in some particular cases, like accidentally stepping out with an unbalanced stride, the human body will adjust naturally to avoid falling down, resulting in unusual changes in the EMG and GRF patterns and possible misjudgment of the classifier. This phenomenon (i.e., the error rate of recognizing RD into $\mathrm{LW}$ ) is higher than the overall error rate and is also found in relevant works [27-29] (a comparison is given in Table II). A potential solution is to enlarge the volume of the training dataset.

Fig. 6 depicts the application of the system developed in this paper under an IoHT architecture. The EMG and GRF information for people who need rehabilitation are detected and processed by front-end detectors; then, the signals are analyzed, and locomotion modes are recognized in end terminals, such as

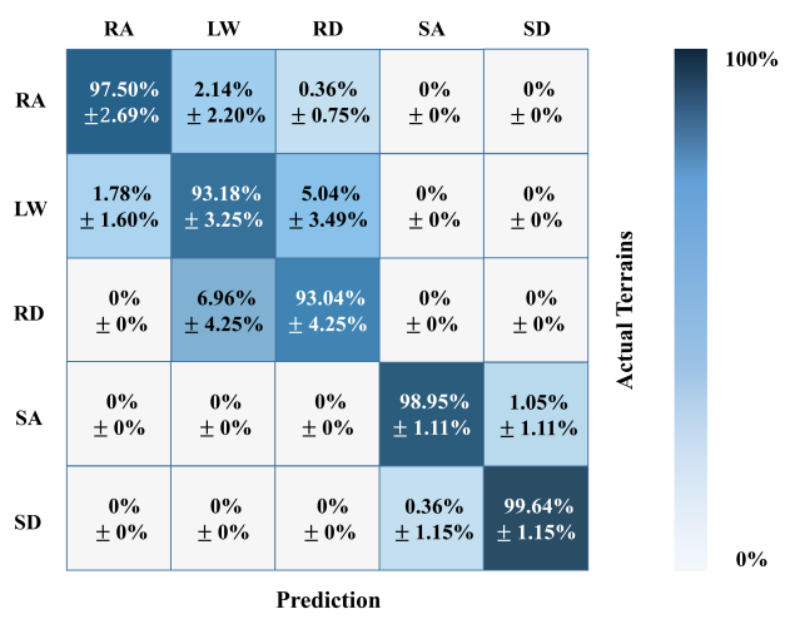

Fig.5. Confusion matrix of the SVM model in recognizing five locomotion modes.

TABLE II. COMPARISON OF ERROR RATE OF RECOGNIZING RD INTO LW AND OVERALL ERROR RATE OF OTHER RELEVANT WORKS AND THIS WORK

\begin{tabular}{|c|c|c|c|c|}
\hline & {$[27]$} & {$[28]$} & {$[29]$} & This work \\
\hline RD-LW error rate & $17.90 \%$ & $2.02 \%$ & $6.79 \%$ & $5.04 \%$ \\
\hline Overall error rate & $10.96 \%$ & $1.65 \%$ & $2.21 \%$ & $3.62 \%$ \\
\hline
\end{tabular}

personal computers or smartphones. Finally, the integrated information is transmitted to remote end terminals. By monitoring changes in the locomotion modes and EMG and GRF data of the user, further analyses can be applied for various purposes, such as disease diagnosis or rehabilitation guidance. The developed IoHT system enables related individuals or institutions to monitor medical data and evaluate therapy performance with the patients at home or in other daily scenarios, which potentially reduces the burdens of both the therapists and the patients and broadens the interaction methods in the rehabilitation field.

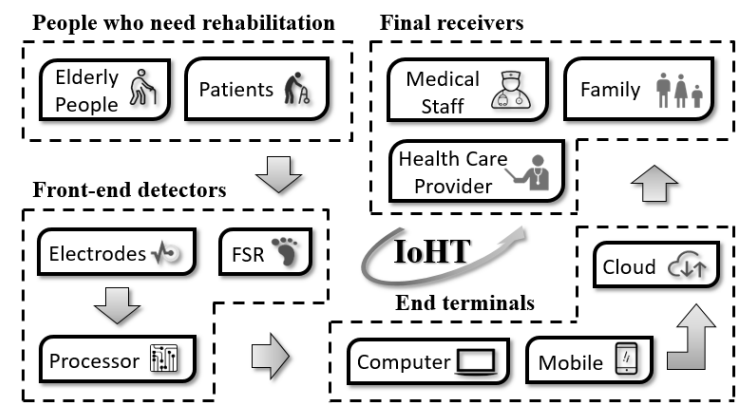

Fig.6. Block diagram for the IoHT architecture consisting of the four elements.

\section{CONCLUSIONS}

Locomotion modes recognition is of significance for inhome rehabilitation. The work presented here demonstrates the design and evaluation of a locomotion mode recognition system utilizing flexible sensors and machine learning technique. A recognition accuracy of $96.38 \%$ is obtained by merely utilizing two EMG and two GRF sensors, which offers a convenient and effective means to reach robust identification accuracy with lower complexity and energy consumption for IoHT based inhome rehabilitation. 


\section{REFERENCES}

[1] S. Nadeau, M. Betschart, and F. Bethoux, "Gait analysis for poststroke rehabilitation: the relevance of biomechanical analysis and the impact of gait speed," Physical Medicine and Rehabilitation Clinics, vol. 24, no. 2, pp. 265-276, 2013.

[2] J. P. Gavin, T. Immins, and T. Wainwright, "Stair negotiation as a rehabilitation intervention for enhancing recovery following total hip and knee replacement surgery," International journal of orthopaedic and trauma nursing, vol. 25, pp. 3-10, 2017.

[3] H. Yano, S. Tamefusa, N. Tanaka, H. Saitou, and H. Iwata, "Gait rehabilitation system for stair climbing and descending," in 2010 IEEE Haptics Symposium, 2010: IEEE, pp. 393-400.

[4] M. Oh-Park, S. Perera, and J. Verghese, "Clinically meaningful change in stair negotiation performance in older adults," Gait \& posture, vol. 36, no. 3, pp. 532-536, 2012.

[5] I. Bisio, A. Delfino, F. Lavagetto, and A. Sciarrone, "Enabling IoT for in-home rehabilitation: Accelerometer signals classification methods for activity and movement recognition," IEEE Internet of Things Journal, vol. 4, no. 1, pp. 135-146, 2016.

[6] S. Jeong, K. Kondo, N. Shiraishi, and Y. Inoue, "An evaluation of the quality of post-stroke rehabilitation in Japan," Clinical Audit, vol. 2, p. $59,2010$.

[7] J. J. Kraal, M. E. Van den Akker-Van Marle, A. Abu-Hanna, W. Stut, N. Peek, and H. M. Kemps, "Clinical and cost-effectiveness of homebased cardiac rehabilitation compared to conventional, centre-based cardiac rehabilitation: Results of the FIT@ Home study," European journal of preventive cardiology, vol. 24, no. 12, pp. 1260-1273, 2017.

[8] J. P. Bettger et al., "Effects of Virtual Exercise Rehabilitation In-Home Therapy Compared with Traditional Care After Total Knee Arthroplasty: VERITAS, a Randomized Controlled Trial," JBJS, vol. 102, no. 2, pp. 101-109, 2020.

[9] Z. Moore, C. Sifferman, S. Tullis, M. Ma, R. Proffitt, and M. Skubic, "Depth Sensor-Based In-Home Daily Activity Recognition and Assessment System for Stroke Rehabilitation," in 2019 IEEE International Conference on Bioinformatics and Biomedicine (BIBM), 2019: IEEE, pp. 1051-1056.

[10] F. Horak, L. King, and M. Mancini, "Role of body-worn movement monitor technology for balance and gait rehabilitation," Physical therapy, vol. 95, no. 3, pp. 461-470, 2015.

[11] V. Bianchi, M. Bassoli, G. Lombardo, P. Fornacciari, M. Mordonini, and I. De Munari, "IoT Wearable Sensor and Deep Learning: An Integrated Approach for Personalized Human Activity Recognition in a Smart Home Environment," IEEE Internet of Things Journal, vol. 6, no. 5, pp. 8553-8562, 2019.

[12] E. Kańtoch, "Human activity recognition for physical rehabilitation using wearable sensors fusion and artificial neural networks," in 2017 Computing in Cardiology (CinC), 2017: IEEE, pp. 1-4.

[13] J. Qi, P. Yang, A. Waraich, Z. Deng, Y. Zhao, and Y. Yang, "Examining sensor-based physical activity recognition and monitoring for healthcare using Internet of Things: A systematic review," Journal of biomedical informatics, vol. 87, pp. 138-153, 2018.

[14] G. Ligorio and A. M. Sabatini, "A novel Kalman filter for human motion tracking with an inertial-based dynamic inclinometer," IEEE Transactions on Biomedical Engineering, vol. 62, no. 8, pp. 2033-2043, 2015.

[15] A. J. Young, A. M. Simon, and L. J. Hargrove, "A Training Method for Locomotion Mode Prediction Using Powered Lower Limb Prostheses,"
IEEE Transactions on Neural Systems and Rehabilitation Engineering, vol. 22, no. 3, pp. 671-677, 2014, doi: 10.1109/TNSRE.2013.2285101.

[16] D. Joshi and M. E. Hahn, "Terrain and Direction Classification of Locomotion Transitions Using Neuromuscular and Mechanical Input," (in English), Annals of Biomedical Engineering, Article vol. 44, no. 4, pp. 1275-1284, Apr 2016, doi: 10.1007/s10439-015-1407-3.

[17] S. Kyeong, W. Shin, M. Yang, U. Heo, J.-r. Feng, and J. Kim, "Recognition of walking environments and gait period by surface electromyography," Frontiers of Information Technology \& Electronic Engineering, 2019.

[18] L. Ming, Z. Fan, and H. H. Helen, "An Adaptive Classification Strategy for Reliable Locomotion Mode Recognition," Sensors (Basel, Switzerland), vol. 17, no. 9, 2017.

[19] H. Huang, F. Zhang, L. J. Hargrove, Z. Dou, D. R. Rogers, and K. B. Englehart, "Continuous locomotion-mode identification for prosthetic legs based on neuromuscular-mechanical fusion," IEEE Transactions on Biomedical Engineering, vol. 58, no. 10, pp. 2867-2875, 2011.

[20] S. Day, "Important factors in surface EMG measurement," Bortec Biomedical Ltd publishers, pp. 1-17, 2002.

[21] H. J. Hermens et al., "European recommendations for surface electromyography," Roessingh research and development, vol. 8, no. 2, pp. 13-54, 1999.

[22] A. Dutta, B. Khattar, and A. Banerjee, "Nonlinear Analysis of Electromyogram Following Neuromuscular Electrical StimulationAssisted Gait Training in Stroke Survivors," in Converging Clinical and Engineering Research on Neurorehabilitation: Springer, 2013, pp. 53-57.

[23] A. Burden, "How should we normalize electromyograms obtained from healthy participants? What we have learned from over 25 years of research," (in English), Journal of Electromyography and Kinesiology, Review vol. 20, no. 6, pp. 1023-1035, Dec 2010, doi: 10.1016/j.jelekin.2010.07.004.

[24] J. W. Wannop, J. T. Worobets, and D. J. Stefanyshyn, "Normalization of ground reaction forces, joint moments, and free moments in human locomotion," Journal of applied biomechanics, vol. 28, no. 6, pp. 665676, 2012.

[25] R. H. Chowdhury, M. B. I. Reaz, M. A. B. Ali, A. A. A. Bakar, K. Chellappan, and T. G. Chang, "Surface Electromyography Signal Processing and Classification Techniques," (in English), Sensors, Review vol. 13, no. 9, pp. 12431-12466, Sep 2013, doi: 10.3390/s130912431.

[26] A. Subasi, "Classification of EMG signals using PSO optimized SVM for diagnosis of neuromuscular disorders," Computers in biology and medicine, vol. 43, no. 5, pp. 576-586, 2013.

[27] T. Afzal, K. Iqbal, G. White, and A. B. Wright, "A method for locomotion mode identification using muscle synergies," IEEE Transactions on Neural Systems and Rehabilitation Engineering, vol. 25, no. 6, pp. 608-617, 2016.

[28] Y. Long et al., "PSO-SVM-based online locomotion mode identification for rehabilitation robotic exoskeletons," Sensors, vol. 16, no. 9 , p. 1408,2016

[29] C. Gong, D. Xu, Z. Zhou, N. Vitiello, and Q. Wang, "Real-Time OnBoard Recognition of Locomotion Modes for an Active Pelvis Orthosis," in 2018 IEEE-RAS 18th International Conference on Humanoid Robots (Humanoids), 2018: IEEE, pp. 346-350. 